\title{
ON PROJECTIVE CHARACTERS OF PRIME DEGREE
}

\author{
by R. J. HIGGS
}

(Received 22 March, 1990)

All groups $G$ considered in this paper are finite and all representations of $G$ are defined over the field of complex numbers. The reader unfamiliar with projective representations is referred to [9] for basic definitions and elementary results.

0. Introduction. Let $\operatorname{Proj}(G, \alpha)$ denote the set of irreducible projective characters of a group $G$ with cocycle $\alpha$. In a previous paper [3] the author showed that if $G$ is a $(p, \alpha)$-group, that is the degrees of the elements of $\operatorname{Proj}(G, \alpha)$ are all powers of a prime number $p$, then $G$ is solvable. However Isaacs and Passman in [8] were able to give structural information about a group $G$ for which $\xi(1)$ divides $p^{e}$ for all $\xi \in \operatorname{Proj}(G, 1)$, where 1 denotes the trivial cocycle of $G$, and indeed classified all such groups in the case $e=1$. Their results rely on the fact that $G$ has a normal abelian $p$-complement, which is false in general if $G$ is a $(p, \alpha)$-group; the alternating group $A_{4}$ providing an easy counter-example for $p=2$.

The aim of this paper is to at least give a full classification of $p$-groups $G$ whose irreducible projective characters with cocycle $\alpha$ all have degree $p$. In Section 1 we shall show that a $(p, \alpha)$-group $G$ which does not possess a normal abelian $p$-complement may be considered unusual, and we shall assume thereafter that $G$ does have such a complement. Under this assumption Isaacs and Passman's results for ordinary characters still hold for projective characters, and our interest is focussed on the necessary changes in the corresponding proofs.

In Section 2 we obtain the following theorem which is the exact analogue of Theorem II of [8].

THEOREM 1. Let $p$ be an odd prime number, and $G$ be a group with a normal abelian p-complement. Then every irreducible projective character of $G$ with cocycle $\alpha$ has degree dividing $p$ if and only if

(i) $G$ is abelian and the cohomology class of $\alpha$ is trivial; or

(ii) $G$ has an abelian normal subgroup $A$ with the cohomology class of $\alpha_{A}$ trivial of minimal index $p$; or

(iii) $G / U$ is a group of order $p^{3}$ and exponent $p$, where $U$ denotes the set of $\alpha$-regular elements of $G$ contained in the centre $Z(G)$ of $G$.

The case $p=2$ is exceptional and is dealt with in Section 3 . Let $C_{n}$ and $D_{n}$ denote the cyclic group of order $n$ and the dihedral group of order $2 n$ respectively. Then with notation as above our results culminate in the following theorem.

Theorem 2. Let $p=2$, and $G$ be a group with a normal abelian 2-complement. Suppose every irreducible projective character of $G$ with cocycle $\alpha$ has degree dividing 2. Then $G$ satisfies (i), (ii), or (iii) of Theorem 1 or

(iv) $U=Z(G)$ and $G / U \cong \mathrm{C}_{2} \times \mathrm{C}_{2} \times \mathrm{C}_{2} \times \mathrm{C}_{2}$, or $D_{4} \times \mathrm{C}_{2}$, or

$$
R=\left\langle x, y, z: x^{4}=y^{2}=z^{2}=1, x y=y x, y z=z y, x z=z x^{-1} y\right\rangle .
$$

Glasgow Math. J. 33 (1991) 311-321. 
1. Prime power degrees. Let $[\alpha]$ denote the cohomology class of the cocycle $\alpha$ in the Schur multiplier $M(G)$ of the group $G$. We start by noting that the degrees of projective representations are unaffected under projective equivalence, so that if $G$ is a $(p, \alpha)$-group then it is also a $(p, \beta)$-group for $[\beta]=[\alpha]$. Thus in what follows it is no loss to assume that the cocycle $\alpha$ under consideration is a class-preserving cocycle, such that $o([\alpha])=n$ if and only if $\alpha^{n}$ is the trivial cocycle of $G$. We also state and use without further reference the fact that $o([\alpha])$ divides $\xi(1)$ for all $\xi \in \operatorname{Proj}(G, \alpha)$. Finally for the remainder of this paper let $p$ be a fixed prime number.

Definition 1.1. A group $G$ is said to have p.r.x. $(e, \alpha)$ (projective representation exponent $e$ ) if there exists a cocycle $\alpha$ of $G$ such that $\xi(1)$ divides $p^{e}$ for all $\xi \in \operatorname{Proj}(G, \alpha)$.

For convenience we quote, summarize, and generalize $2.4,2.7$ and 2.8 of [8], and $2.2,2.3,2.4$, and Theorem $B$ of [3], the proofs where needed can easily be derived from those given in the relevant paper.

LemMA 1.2. Let $G$ have p.r.x.(e, $\alpha), N \unlhd G, \xi \in \operatorname{Proj}\left(N, \alpha_{N}\right)$, and $I_{G}(\zeta)$ denote the inertia group of $\zeta$ in $G$. Then

(i) $N$ has p.r.x. $\left(e, \alpha_{N}\right)$;

(ii) if $G / N$ is non-abelian, then $N$ has p.r.x. $\left(e-1, \alpha_{N}\right)$;

(iii) $I_{G}(\zeta) / N$ has p.r.x.(e, $\left.\beta\right)$ for some cocycle $\beta$ of $I_{G}(\zeta) / N$, and $\left[G: I_{G}(\zeta)\right]$ divides $p^{e}$.

Lemma 1.3. Let $N \unlhd G$ with $G / N$ a p-group. Suppose $G$ has p.r.x.(e, $\alpha)$ and $N$ has p.r.x. $\left(e-1, \alpha_{N}\right)$. Then $F$ has p.r.x. $\left(e-1, \alpha_{N}\right)$, where $F$ is the inverse image in $G$ of the Frattini subgroup of $G / N$.

Lemma 1.4. Let $G$ have p.r.x. $(e, \alpha), L \leq G$ such that $[G: L]$ is coprime to $p$, and $\zeta \in \operatorname{Proj}\left(L, \alpha_{L}\right)$. Then $\zeta$ extends to $G$.

Theorem 1.5. Let $G$ have p.r.x. $(e, \alpha)$. Then $G$ is solvable and has abelian Hall $p^{\prime}$-subgroups.

Lemma 1.6. Let $G$ have p.r.x.(e, $\alpha)$ and suppose $G$ has a normal abelian $p$ complement. Let $H \unlhd K \leq G$ with $K / H$ an abelian group of order coprime to $p$. Then

(i) $K$ has p.r.x. $\left(e, \alpha_{K}\right)$;

(ii) if $H$ has p.r.x. $\left(f, \alpha_{H}\right)$, then $K$ has p.r.x. $\left(f, \alpha_{K}\right)$.

Our next aim is to relate the results on projective and ordinary representation exponents, the following proposition providing the crucial link.

Proposition 1.7. Let $G$ have p.r.x. $(e, \alpha), p^{a}=\min \{\xi(1): \xi \in \operatorname{Proj}(G, \alpha)\}$, and suppose $G$ has a normal abelian $p$-complement. Then $G$ has p.r.x. $\left(e+a, \alpha^{n}\right)$ for any integer $n$.

Proof. Let $S$ be a Sylow $p$-subgroup of $G$, and $\zeta \in \operatorname{Proj}\left(S, \alpha_{S}\right)$ of minimum degree. Then $\zeta(1)=p^{a}$ by Proposition 1 of [2]. Now since $S$ is a $P M$-group there exists a subgroup $T$ of $S$ with $[S: T]=p^{a}$ and $\lambda \in \operatorname{Proj}\left(T, \alpha_{T}\right)$ with $\lambda^{S}=\zeta$.

Let $N$ be the normal abelian $p$-complement of $G$, then by 1.6(i) $T N$ has p.r.x. $\left(e, \alpha_{T N}\right)$, and so by $1.4 \lambda$ extends to $\mu \in \operatorname{Proj}\left(T N, \alpha_{T N}\right)$. Thus $\left[\alpha_{T N}\right]=[1]$, so that $G$ 
is a $\left(p, \alpha^{n}\right)$-group for any integer $n$ by Theorem 2 of $[11]$, and since $[G: T N]=p^{a}$ it follows that $G$ has p.r.x. $\left(e+a, \alpha^{n}\right)$.

The above result allows us to generalize Theorem $I$ of [8] as follows.

THEOREM 1.8. Let $G$ have p.r.x. $(e, \alpha)$ and suppose $G$ has a normal abelian p-complement. Then $G$ has a series of subgroups

$$
A_{0} \unlhd A_{1} \unlhd \ldots \unlhd A_{e}=G,
$$

such that $A_{0}$ is abelian, $\left[\alpha_{A_{0}}\right]=[1]$, and $A_{i} / A_{i-1}$ is an elementary abelian p-group with not more than $2(i+a)+1$ generators; where $p^{a}=\min \{\xi(1): \xi \in \operatorname{Proj}(G, \alpha)\}$. Hence $G$ has an abelian subgroup $A_{0}$ with $\left[\alpha_{A_{0}}\right]=[1]$ whose index divides $p^{e(e+2 a+2)}$.

Proof. We proceed by induction on $e$, noting that if $A$ is a subnormal subgroup of $G$ then $\min \left\{\zeta(1): \zeta \in \operatorname{Proj}\left(A, \dot{\alpha}_{A}\right)\right\}$ divides $p^{a}$. Thus it suffices to prove that there exists a normal subgroup $A_{e-1}$ of $G$, such that $A_{e-1}$ has p.r.x. $\left(e-1, \alpha_{A_{e-1}}\right)$ and $G / A_{e-1}$ is an elementary abelian $p$-group of order $\leq p^{2(e+a)+1}$.

Suppose $G$ is abelian. Then $\operatorname{Proj}(G, \alpha)$ all have the same degree. Let $U$ denote the set of $\alpha$-regular elements of $G$ and $\xi \in \operatorname{Proj}(G, \alpha)$. Then $U$ has p.r.x. $\left(0, \alpha_{U}\right), \xi_{U}=\xi(1) \lambda$ for some $\lambda \in \operatorname{Proj}\left(U, \alpha_{U}\right)$, and $\xi(g)=0$ for $g \in G-U$. Thus $\left[\alpha_{U}\right]=[1]$ and $[G: U]=p^{a^{2}}$. If $U=G$ there is nothing to prove, whereas if $U<G$ we may let $A_{e-1}$ be a maximal subgroup of $G$ with $U \leq A_{e-1}<G$. Then $\left[G: A_{e-1}\right]=p$, and $A_{e-1}$ has p.r.x. $\left(e-1, \alpha_{A_{e-1}}\right)$ from above.

Suppose $G$ is non-abelian. Let $N \triangleleft G$ be maximal such that $G / N$ is non-abelian and set $\bar{G}=G / N$. Let $H$ be the inverse image in $G$ of the normal abelian $p$-complement of $\bar{G}$. Then $N$ has p.r.x. $\left(e-1, \alpha_{N}\right)$ by 1.2 , and $H$ has p.r.x. $\left(e-1, \alpha_{H}\right)$ by 1.6 .

Case 1: $\bar{H}=H / N$ is a non-trivial subgroup of $\bar{G}$.

In this case $\bar{G}$ is a Frobenius group with an abelian Frobenius complement, $\bar{G}^{\prime}$ is the Frobenius kernel and is an elementary abelian group. It follows that $\bar{G}^{\prime} \leq \bar{H}$, and $\bar{H}$ is both a maximal abelian normal subgroup of $\bar{G}$ and a $q$-group for some prime $q \neq p$. By 2.9 of [8] and 12.3 of [7] we conclude that $\bar{H}=\bar{G}^{\prime}$. Now it follows from 12.4 of [7] that if $\zeta \in \operatorname{Proj}\left(H, \alpha_{H}\right)$, then $[G: H] \zeta(1)$ is the degree of an element of $\operatorname{Proj}(G, \alpha)$. Thus $[G: H] \leq p^{e}$.

Finally let $A_{e-1}$ be the inverse image in $G$ of the Frattini subgroup of $G / H$. Then by $1.3 A_{e-1}$ has p.r.x. $\left(e-1, \alpha_{A_{e-1}}\right)$, and $G / A_{e-1}$ is an elementary abelian $p$-group of order $\leq p^{e}$.

Case 2: $\bar{G}$ is a $p$-group.

In this case let $A_{e-1}$ be the inverse image in $G$ of the Frattini subgroup of $\bar{G}$. Then by 1.3 $A_{e-1}$ has p.r.x. $\left(e-1, \alpha_{A_{e-1}}\right)$. Also by Case 2 p. 451 of $[8]$ and $1.7, G / A_{e-1}$ is an elementary abelian $p$-group of order $\leq p^{2(e+a)+1}$.

We now state a derivative of the theorem which we shall use in Section 2.

COROllary 1.9. Let $G$ have p.r.x. $(e, \alpha)$, and suppose $G$ has a normal abelian p-complement and an abelian Sylow p-subgroup. Then $G$ has a series of subgroups

$$
A_{0} \unlhd A_{1} \unlhd \ldots \unlhd A_{e}=G,
$$

such that $A_{0}$ is abelian, $\left[\alpha_{A_{0}}\right]=[1]$, and $A_{i} / A_{i-1}$ is an elementary abelian p-group with not more than $i$ generators. Hence $G$ has an abelian subgroup $A_{0}$ with $\left[\alpha_{A_{0}}\right]=[1]$ whose index divides $p^{e(e+1) / 2}$. 
Proof. The result follows from the proof of 1.8 by strengthening the inductive hypothesis and noting that Case 2 of that proof cannot occur.

Let $G$ have p.r.x. $(e, \alpha)$. Then the previous two results have relied on the assumption that $G$ has a normal abelian $p$-complement. The last two results in this section deal with the circumstances under which this assumption is tenable.

It is convenient in what follows to call a group $H$ an $F_{i}$-group if $H$ is a Frobenius group of order $p^{i} q$, where $q$ is a prime number such that $q$ divides $p^{i}-1$, and the Frobenius kernel of $H$ is an elementary abelian group of order $p^{i}$.

Lemma 1.10. Let $G$ be an $F_{i}$-group with Frobenius kernel $S$, and suppose $G$ is a $(p, \alpha)$-group. Then $\operatorname{Proj}(G, \alpha)$ consists of q-extensions of the unique element of $\operatorname{Proj}\left(S, \alpha_{s}\right)$.

Proof. Let $\zeta \in \operatorname{Proj}\left(S, \alpha_{S}\right)$. Then it follows from 12.4 of [7] that $\zeta(1)^{2}=|S|$. Also $\zeta$ extends to $G$ by 1.4 .

To illustrate the above lemma we may take $p=2$ and then $A_{4}$ is the only example of an $F_{2}$-group which also has p.r.x. $(1, \alpha)$, for $[\alpha]$ the non-trivial element of $M\left(A_{4}\right)$. We also note for future reference that if $\alpha$ is a cocycle of a group $G$ such that $|\operatorname{Proj}(G, \alpha)|=1$, then $G$ is said to be of $\alpha$-central type.

If $N$ is a normal subgroup of a group $G$, we shall let inf denote the inflation homomorphism from $M(G / N)$ into $M(G)$.

Proposition 1.11. Let $G$ be a group of minimal order which has p.r.x. $(e, \alpha)$ but does not possess a normal abelian p-complement. Let $K \triangleleft G$ be maximal such that $G / K$ is non-abelian. Then $G / K$ is an $F_{i}$-group for $1 \leq i \leq 2 a$, where $p^{a}=\min \{\xi(1): \xi \in$ $\operatorname{Proj}(G, \alpha)\}$.

In particular if $a=1$, then either $G / K$ is an $F_{2}$-group which has p.r.x. $(1, \beta)$ for some cocycle $\beta$ of $G / K$ with $\inf ([\beta])=[\alpha]$, or $G / K$ is an $F_{1}$-group.

Proof. Let $L / K=(G / K)^{\prime}$. Then by 12.3 and 12.4 of [7] and 1.5, all the non-linear irreducible ordinary characters of $G / K$ have equal degree $f, G / K$ is a Frobenius group with an abelian Frobenius complement of order $f$ and an elementary abelian Frobenius kernel $L / K$; also if $\zeta \in \operatorname{Proj}\left(L, \alpha_{L}\right)$ then $V(\zeta) \leq K$ and $[L: K]$ divides $\zeta(1)^{2}$, where $V(\zeta)$ denotes the vanishing-off subgroup of $\zeta$. Thus $[L: K]$ divides $p^{2 a}$ and $f$ divides $[L: K]-1$. Now let $T$ be a maximal normal subgroup of $G$ containing $L$. Then by $1.2, T$ and hence $T / K$ have normal abelian $p$-complements. Since $f$ is coprime to $p$, it follows that $T=L$ and $f=q$ for some prime $q=[G: L]$.

It remains to show that if $a=1$ and $G / K$ is an $F_{2}$-group, then $G / K$ has p.r.x. $(1, \beta)$ for some cocycle $\beta$ of $G / K$ with $\inf ([\beta])=[\alpha]$. Let $\xi \in \operatorname{Proj}(G, \alpha)$ with $\xi(1)=p$. Then $\xi_{L}=\zeta$ for some $\zeta \in \operatorname{Proj}\left(L, \alpha_{L}\right)$, since $[G: L]=q$. However, the inner product $\left\langle\zeta_{K}, \zeta_{K}\right\rangle=p^{2}$ since $V(\zeta) \leq K$, and so $\xi_{K}=p \lambda$ for some $\lambda \in \operatorname{Proj}\left(K, \alpha_{K}\right)$. Thus $I_{G}(\lambda)=G$, and the desired result follows from 1.2 and 1.10 .

2. Prime degree. Throughout Section 2 we shall assume that $G$ is a group having p.r.x. $(1, \alpha)$ and a normal abelian $p$-complement.

Definition 2.1. A subgroup $A$ of $G$ is said to be special if

(i) $A$ is an abelian normal subgroup of $\mathrm{G}$ such that $\left[\alpha_{A}\right]=[1]$;

(ii) $G / A$ is an elementary abelian $p$-group;

(iii) if $A<B$, then either $B$ is non-abelian or $B$ is abelian but $\left[\alpha_{B}\right] \neq[1]$. 
It is convenient to call the special subgroup $A$ more-special if $A<B$ implies $B$ is non-abelian, and less-special otherwise. We note that with the assumptions made above 1.8 yields a special subgroup of $G$ of index dividing $p^{5}$.

Lemma 2.2. Suppose $p$ is odd, and let $A$ be a special subgroup of $G$. Then each element $a \in A$ has $C_{\alpha}(a)=A$ or $G$, where $C_{\alpha}(a)=\left\{x \in C_{G}(a): \alpha(a, x)=\alpha(x, a)\right\}$.

Proof. If $a \in A$, then $A \leq C_{\alpha}(a)$ since $\left[\alpha_{A}\right]=[1]$. Thus the result is trivial if $[G: A] \leq p$.

Now suppose $a \in A$ with $A<C_{\alpha}(a)<G$. Choose $x \in C_{\alpha}(a)-A$ and $y \in G-C_{\alpha}(a)$, and set $K=\langle A, x, y\rangle$. By (ii) of 2.1 it is clear that $[K: A]=p^{2}$. Since $x \notin A, B=\langle A, x\rangle$ is either non-abelian or is abelian with $\left[\alpha_{B}\right] \neq[1]$. In either case there exists $b \in A$ with $x \notin C_{\alpha}(b)$. Let $\bar{z}$ denote the element $z \in G$ viewed as an element of the twisted group algebra $\mathbb{C} G_{\alpha}$. Then $u=(\bar{x})^{-1}(\bar{b})^{-1} \bar{x} \bar{b}$ and $v=(\bar{y})^{-1}(\bar{a})^{-1} \bar{y} \bar{a}$ are non-identity elements of $\mathbb{C} A_{\alpha_{A}}$. Now by working in $\mathbb{C} A_{\alpha_{A}}$ the proof of 3.3 of [8] carries over to our situation to give a contradiction, provided that $\overline{1}+u v \neq u+v$. However writing $u=c \bar{w}$ and $v=k \bar{z}$, where $w=[x, b], z=[y, a]$ and $c, k$ are $p$ th roots of unity; we have that $\overline{1}+u v=u+v$ if and only if $z^{-1}=w=z \neq 1, k=-c, p=2$, and $\alpha(w, w)=1$.

We note in the context of 2.2 that if $A$ is more-special then replacing $C_{\alpha}(a)$ by $C_{G}(a)$ in the proof yields that $C_{G}(a)=A$ or $G$ for each $a \in A$, provided that $\overline{1}+u v \neq u+v$. This observation coupled with the lemma allows us to describe the $\alpha$-regular elements of a special subgroup of $G$. Let $U=\{z \in Z(G): z$ is $\alpha$-regular $\}$, the reader may refer to [12] for various characterizations of $U$.

Lemma 2.3. Let $A$ be a special subgroup of $G$ and suppose $[G: A] \neq p$. Then $A \cap Z(G)=U$. In particular if $A$ is more-special then $U=Z(G)$.

Proof. Clearly $U<A$ by definition of $A$, and also $Z(G)<A$ if $A$ is more-special. Let $K=A \cap Z(G)$. Then by 2.2 (and its proof in the case $p=2$ ) each $a \in K$ has $C_{\alpha}(a)=A$ or $G$. Let $\lambda \in \operatorname{Proj}\left(K, \alpha_{K}\right)$, then $\lambda^{x}=\lambda$ if and only if $\alpha(a, x)=\alpha(x, a)$ for all $a \in K$. Thus $I_{G}(\lambda)=\bigcap_{a \in K} C_{\alpha}(a)=A$ or $G$. If $I_{G}(\lambda)=A$, then $\lambda$ has $[G: A]$ conjugates. Since $G$ has p.r.x. $(1, \alpha)$ we conclude that $K=U$.

For the rest of this section we shall assume that if $A$ is a special subgroup of $G$ then $C_{\alpha}(a)=A$ or $G$ for each $a \in A$, we shall also assume that $C_{G}(a)=A$ or $G$ for each $a \in A$ when $A$ is more-special. Of course these assumptions are certainly valid for $p \neq 2$ by 2.2 , and will be discussed in detail for $p=2$ in Section 3 .

Proposition 2.4. Let $A$ be a special subgroup of $G$ and suppose $[G: A] \neq p$. Then every element of $A$ is $\alpha$-regular if $A$ is more-special, whereas the elements of $U$ are the $\alpha$-regular elements of $A$ if $A$ is less-special.

Proof. Suppose $a \in A-U$. Then $C_{\alpha}(a)=A$. However using 2.3 we have that $C_{G}(a)=A$ if $A$ is more-special, whereas $A<C_{G}(a)$ if $A$ is less-special.

We next show that $U$ has index $p$ in a special $\operatorname{subgroup} A$ with $[G: A]>p$. This will enable us to classify $G / U$ in subsequent results.

Proposition 2.5. Let $A$ be a special subgroup of $G$ of index $p^{t}$.

(a) If $t>1$ or $t=1$ and $A$ is less-special, then $[A: U]=p$.

(b) If $t=1$ and $A$ is more-special, then $p|Z(G)|\left|G^{\prime}\right|=|G|$. 
Proof. Suppose first that $A$ is less-special. Let $B$ be an abelian group with $[B: A]=p$. Then $\left[\alpha_{B}\right] \neq[1]$, so that all elements of $\operatorname{Proj}\left(B, \alpha_{B}\right)$ have degree $p$ and vanish on $B-A$. Let $T$ denote the set of $\alpha_{B}$-regular elements of $B$. Then since $B$ is abelian it follows as in the proof of 1.8 that $[A: T]=p$, and all elements of $\operatorname{Proj}\left(B, \alpha_{B}\right)$ are non-zero exactly on $T$. From 2.4 (or trivially if $B=G$ ) we conclude that $T=U$, since every element of $\operatorname{Proj}(G, \alpha)$ restricts irreducibly to $B$.

Now suppose $A$ is more-special. Suppose also $t>1$. Then by 1.8 of [3], 2.3, 2.4, and the proof of 3.5 of $[8]$ we obtain the equation

$$
|U|\left(p^{t}-1\right)=|A|\left(p^{t-1}-1\right)+k\left(p^{t}-p^{t-1}\right),
$$

where $k$ is the number of $G$-invariant elements of $\operatorname{Proj}\left(A, \alpha_{A}\right)$. Suppose $k=0$, then $p^{t-1}-1$ divides $p^{t}-1$, which is impossible. Thus there exists a $G$-invariant element of $\operatorname{Proj}\left(A, \alpha_{A}\right)$. Let $Q$ be the Sylow $p$-subgroup of $A$, then it follows from 1.2 that $G / Q$ has p.r.x. $(1, \beta)$ for some cocycle $\beta$ of $G / Q$ such that $\inf ([\beta])=[\alpha]$. The proof of 3.4 of $[8]$, and 1.9 now yield that the normal abelian $p$-complement of $G$ is central. Thus since $[A: U]$ is a power of $p$, we obtain from (1) that $[A: U]=p$. Suppose now $t=1$. Then $G$ is non-abelian and has p.r.x. $(1,1)$, so that $(\mathrm{b})$ is just $3.5(\mathrm{~b})$ of $[8]$. (Note: the proof of 3.5(b) of $[8]$ is independent of the assumption that $G$ is a $p$-group.)

Lemma 2.6. Suppose $A$ is a special subgroup of $G$ of minimal index $p^{\prime}$, where $t>1$ or $t=1$ and $A$ is less-special. Then

(i) $G / U$ has exponent $p$;

(ii) $G / T$ is abelian for any $T$ with $U<T \unlhd G$;

(iii) if $G / U$ is non-abelian, then $A / U=(G / U)^{\prime}$ is the unique minimal normal subgroup of $G / U$.

Proof. (i) Suppose there exists an element $x$ of order $p^{2}$ in $G / U$. Then $x^{p} \in A-U$, so that $A$ is a proper subgroup of $\langle x, U\rangle$ since $[A: U]=p$ by 2.5 . But $\langle x, U\rangle$ is a special subgroup of $G$, contrary to the minimality of $[G: A]$.

(ii) Suppose there exists $T \varangle G$ with $U<T$ such that $G / T$ is non-abelian. Then $T$ is abelian with $\left[\alpha_{T}\right]=[1]$ by 1.2 . Let $F$ be the inverse image in $G$ of the Frattini subgroup of $G / T$. Then $F$ is abelian with $\left[\alpha_{F}\right]=[1]$ by 1.3 , but $[G: F]<[G: A]$ by 2.5 , a contradiction.

(iii) If $G / U$ is non-abelian, then by (ii) $U$ is a maximal normal subgroup of $G$ such that $G / U$ is non-abelian. Thus $(G / U)^{\prime}=A / U$ is the unique minimal normal subgroup of G.

We can now prove Theorem 1, noting that the proof we shall give still holds in the case $p=2$, provided that all special subgroups of $G$ of minimal index satisfy the assumptions of this section.

Proof of Theorem 1. Let $A$ be a special subgroup of $G$ of minimal index $p^{t}$, so that $t \leq 5$ by 1.8 . If $t=0$ or 1 , then $G$ satisfies (i) or (ii) respectively. Also if $[\alpha]=[1]$, then $G$ satisfies (i), (ii) or (iii) by Theorem II of [8]. So suppose $t>1$ and $[\alpha] \neq[1]$. Then by 2.5 and 2.6, $G / U$ has exponent $p$ and order $p^{t+1}$, and if $G / U$ is non-abelian (so $p \neq 2$ ) it must be an extra-special $p$-group of order $p^{3}$ or $p^{5}$, since in this case $Z(G / U)$ is cyclic.

Stage 1: If $t \geq 3, G / U$ is not elementary abelian.

Let $x \in G-U$. Then $\langle x, U\rangle$ is a special subgroup of $G$, so that $C_{\alpha}(x)=A$ from 2.2 . Thus every $\alpha$-regular conjugacy class of $G$ contains either 1 or $p^{t}$ elements. Let $u=|U|$ 
and $[r]$ temporarily denote the integral part of the real number $r$. Then $G$ has at most $u+\left[(|G|-u) / p^{t}\right]=u+\left[u\left(p^{t+1}-1\right) / p^{t}\right] \alpha$-regular conjugacy classes. But we know that $G$ has exactly $|G| / p^{2}=u p^{t-1} \alpha$-regular conjugacy classes, since $G$ has p.r.x. $(1, \alpha)$ and $[\alpha] \neq[1]$. So we certainly require that $1+p^{-t}\left(p^{t+1}-1\right) \leq p^{t-1}$ i.e. $p^{t}-1 \geq p^{t+1}\left(p^{t-2}-1\right)$, which is clearly impossible for $t \geq 3$.

Stage 2: $G / U$ is not an extra-special group of order $p^{5}$.

Let $\bar{G}=G / U$ and $\bar{Z}=A / U$. Then from the proof of 3.3.6 of [10], inf: $M(\bar{G} / \bar{Z}) \rightarrow$ $M(\bar{G})$ is a surjection with kernel of order $p$. Let $\beta$ be a cocycle of $\bar{G} / \bar{Z}$ such that $\operatorname{ker}($ inf $)=\langle[\beta]\rangle$ and $\beta^{p}=1$. Then by V.16.14 of [6] only the identity element of $\bar{G} / \bar{Z}$ is $\beta^{i}$-regular for $1 \leq i \leq p-1$. Now each $\xi \in \operatorname{Proj}(G, \alpha)$ has $\xi_{U}=p \lambda$ for some $\lambda \epsilon$ $\operatorname{Proj}\left(U, \alpha_{U}\right)$. It follows from 1.2 both that there exist cocycles $\bar{\alpha}$ of $G / U$ with $[\bar{\alpha}]$ inflated to $G$ equal to $[\alpha]$, and that $G / U$ has p.r.x. $(1, \bar{\alpha})$ for each such $\bar{\alpha}$. Similarly, since every element of $\bar{Z}$ is $\bar{\alpha}$-regular, there exist cocycles $\beta^{i} \gamma$ for $0 \leq i \leq p-1$ with $\inf ([\gamma])=[\bar{\alpha}]$ for which $\bar{G} / \bar{Z}$ has p.r.x. $\left(1, \beta^{i} \gamma\right)$. We thus require that $\bar{G} / \bar{Z}$ contains exactly $p^{2} \beta^{i} \gamma$-regular elements for each $i$ with $0 \leq i \leq p-1$.

Let $\bar{G} / \bar{Z}=\left\langle x_{1}\right\rangle \times \ldots \times\left\langle x_{4}\right\rangle$, and $\beta\left(x_{i}, x_{j}\right)=\omega^{c_{i j}}$, where $\omega$ is a non-trivial $p$ th root of unity. Let $C$ be the skew-symmetric matrix whose $(i, j)$ th entry is $c_{i j}$ for $j>i$. Then $x_{1}^{b_{1}} \ldots x_{4}^{b_{4}}$ is $\beta$-regular if and only if $C \vec{b}=\overrightarrow{0}$ in $\mathbb{Z}_{p}^{4}$, where $\vec{b}=\left[b_{1}, \ldots, b_{4}\right]^{T}$. Now since no non-trivial element of $\bar{G} / \bar{Z}$ is $\beta$-regular we have that $C$ has rank 4 , and so there exists $M \in \mathrm{GL}(4, p)$ with

$$
M^{T} C M=\left[\begin{array}{rrrr}
0 & 1 & 0 & 0 \\
-1 & 0 & 0 & 0 \\
0 & 0 & 0 & 1 \\
0 & 0 & -1 & 0
\end{array}\right] .
$$

Let $D$ be the matrix constructed from $\gamma$ in the same way, then we require that $M^{T} D M+i M^{T} C M$ has rank 2 for $0 \leq i \leq p-1$. So if

$$
M^{T} D M=\left[\begin{array}{rrrr}
0 & a & b & c \\
-a & 0 & d & e \\
-b & -d & 0 & f \\
-c & -e & -f & 0
\end{array}\right]
$$

we must have that $(a+i)(f+i)+b e-c d=0$ for $0 \leq i \leq p-1$. Setting $i=-a$ gives $b e-c d=0$, and we are left with $(a+i)(f+i)=0$ for $0 \leq i \leq p-1$, which is impossible for $p \geq 3$.

Stages 1 and 2 thus yield that $G / U$ is of type (iii).

The converse statement in the theorem is proved in essentially the same way as that of Theorem II of [8].

As an immediate and perhaps surprising corollary of Theorem 1 we obtain the following stronger version of 1.7 .

Corollary 2.7. Let $p$ be odd and let $G$ have p.r.x.(1, $\alpha)$. Suppose $G$ has a normal abelian p-complement. Then $G$ has p.r.x. $\left(1, \alpha^{n}\right)$ for any integer $n$.

To conclude this section we note that if we allow $p=2$ in the matrix calculations of stage 2 above, then we find that the extra-special 2 -groups $G$ of order 32 have p.r.x. $(1, \alpha)$ 
for 10 cohomology classes out of the 32 in $M(G)$. Since these groups are not classified by Theorem 1 (with $p=2$ ), they provide counter-examples to the results of 2.2 and will be dealt with in the following section.

3. Degree two. In this section we shall deal exclusively with a group $G$ having p.r.x. $(1, \alpha)$ and a normal abelian $p$-complement which is not classified by Theorem 1 . In particular then $p=2$, and we can assume that there exists a special subgroup $A$ of $G$ of minimal index $2^{r}>2$, with an element $a \in A$ such that either $A<C_{\alpha}(a)<G$ or $A$ is more-special and $A<C_{G}(a)<G$. With this notation fixed for the duration of this section we can now prove.

Proposition 3.1. $A$ is more-special and $G / A$ is an elementary abelian group of order 4.

Proof. Let $C(a)$ denote $C_{\alpha}(a)$ or if $A$ is more-special $C_{G}(a)$. Then we may treat the two separate possibilities above simultaneously. Now the proof of 2.2 yields for all $x \in C(a)-A$, all $y \in G-C(a)$, and all $b \in A$ with $x \notin C(b)$, that $z^{-1}=w=z \neq 1$, where $w=[x, b]$ and $z=[y, a]$. If $C(a)=C_{\alpha}(a)$ this gives that $a$ is $\alpha$-regular, and then that $A$ must be more-special, otherwise we can obtain that $z$ or $w=1$ respectively. Thus $A$ is more-special and $C(a)=C_{G}(a)$.

Now for any choice of $y_{1}, y_{2} \in G-C_{G}(a)$ we must have that $\left[y_{1}, a\right]=\left[y_{2}, a\right]$ i.e. $y_{1} y_{2}^{-1} \in C_{G}(a)$, so that $\left[G: C_{G}(a)\right]=2$. By 2.5 of [8] there exists a subgroup $T$ of $G$ of index 2 with $A<T$, such that $T$ has p.r.x. $\left(1, \alpha_{T}\right)$ and $\left[\alpha_{T}\right]=[1]$. Clearly $A$ is a special subgroup of $T$, so that by 3.3 of [8] or $2.2 C_{\alpha_{T}}(a)=C_{T}(a)=A$ or $C_{\alpha_{T}}(a)=C_{T}(a)=T$.

In the former case there exists $g \in G-T$ with $g \in C_{G}(a)$, and since $g^{2} \in T$ we have that $g^{2} \in A$. Thus in this case it follows that $C_{\alpha}(a)=C_{G}(a)=\langle A, g\rangle$ or $C_{\alpha}(a)=A$ and $C_{G}(a)=\langle A, g\rangle$.

In the latter case we conclude by 2.3 that $C_{\alpha}(a)=C_{G}(a)=T$. In this case if $T$ is the unique subgroup of $G$ satisfying 2.5 of [8] then $T$ must be abelian, contrary to the fact that $A$ is more-special. So we may let $S$ be another subgroup of $G$ of index 2 with the same properties as $T$. Then as before $C_{S}(a)=A$ or $S$. Since $S T=G$, we must have that $C_{S}(a)=A$, and the desired results follows as in the first case.

By 3.1 each element $b$ of $A$ is classified into one of four types according to whether 1 . $b \in U=Z(G) ; 2$. $C_{\alpha}(b)=A=C_{G}(b) ; 3 . C_{\alpha}(b)=A$ and $\left[G: C_{G}(b)\right]=2 ; 4 . C_{\alpha}(b)=C_{G}(b)$ and $\left[G: C_{G}(b)\right]=2$. Let $z, r, s$, and $t$ denote respectively the number of elements of each type contained in $A$. Also let $t_{1}, t_{2}$, and $t_{3}$ be the number of elements of type 4 centralized by $T_{1}=\langle A, x\rangle, T_{2}=\langle A, y\rangle$, and $T_{3}=\langle A, x y\rangle$ respectively; where $G / A=\langle A x, A y\rangle$ and $C_{G}(a)=\langle A, x\rangle$. Using this notation we now show.

Proposition 3.2. (i) $G / U$ has order 16 and exponent 2 or 4.

(ii) $G / U$ has p.r.x.(1, $\beta)$ for any cocycle $\beta$ of $G / U$ with $\inf ([\beta])=[\alpha]$.

(iii) $G / U$ is of $\gamma$-central type for some cocycle $\gamma$ of $G / U$ with $\inf ([\gamma])=[1]$.

Proof. We first note by 3.1 that $A$ is a special subgroup of $T_{i}$ of index 2 with respect to the trivial cocycle of $T_{i}$, so that from 3.5 of $[8]|A|=\left|Z\left(T_{i}\right)\right|\left|T_{i}^{\prime}\right|$ for $1 \leq i \leq 3$.

Case 1: $A$ contains an element of type 3.

We may assume for notational convenience that $a$ is of type 3 . Now the number of $x$-invariant elements of $\operatorname{Proj}\left(A, \alpha_{A}\right)$ is 0 , since $a$ is not $\alpha$-regular. Let $\lambda \in \operatorname{Proj}\left(A, \alpha_{A}\right)$, then 
we may assume without loss of generality that $I_{G}(\lambda)=T_{2}$. If this is true for all elements of $\operatorname{Proj}\left(A, \alpha_{A}\right)$ then $T_{2}$ is abelian, contrary to the fact that $A$ is more-special. So there exists $\lambda^{\prime} \in \operatorname{Proj}\left(A, \alpha_{A}\right)$ with $I_{G}\left(\lambda^{\prime}\right)=T_{3}$. Now let $b$ be any type 3 element, then by the arguments above $C_{G}(b)=T_{1}$. So by 1.8 of $[4], 0=z-s+t_{1}$; and similarly by considering $y$ and $x y$, $2 z+t_{2}+t_{3}=|A|=z+r+s+t$, so that $z=r+s+t_{1}$. Thus $r+2 t_{1}=0$, and hence $r=t_{1}=$ $0, z=s$, and $\left|Z\left(T_{1}\right)\right|=2 z$.

We now consider $T_{1}^{\prime}$. We note from the proof of 3.1 that $\left[x^{\prime}, b\right]$ is the same element $w$ of order 2 for all $b \in A-Z\left(T_{1}\right)$ and all $x^{\prime} \in T_{1}-A$. Let $c, d \in A$, then

$$
[c x, d x]= \begin{cases}1, & \text { if } c, d \in Z\left(T_{1}\right) \text { or } c, d \notin Z\left(T_{1}\right) ; \\ w, & \text { otherwise. }\end{cases}
$$

Similar calculations show that $[c x, d]$ or $[c, d x]$ is 1 or $w$, and hence $T_{1}^{\prime}$ is the group of order 2 generated by $w$. Thus $[A: U]=4$.

Case 2: $A$ contains no element of type 3 .

In this case every element of $A$ is $\alpha$-regular, and $a$ is of type 4. As in the proof of 3.1 we have for $c$ a type 4 element that for all $x^{\prime} \in C_{G}(c)-A$, all $y^{\prime} \in G-C_{G}(c)$, and all $b \in A$ with $x^{\prime} \notin C_{G}(b)$, that $z^{-1}=w=z \neq 1$; where $w=\left[x^{\prime}, b\right]$ and $z=\left[y^{\prime}, c\right]$. Suppose $c$ has $C_{G}(c)=T_{2}$, then it follows that for any type 2 element ' $b$ ' that $[y, b]=[x, b]$, so that $x y^{-1} \in C_{G}(b)=A$, a contradiction. We obtain a similar contradiction if we assume that $C_{G}(c)=T_{3}$. Thus either $r=0$, or all type 4 elements $c$ have $C_{G}(c)=T_{1}$.

Now $|A|=z+r+t$, and $A$ contains $z+\frac{r}{4}+\frac{t}{2}$ conjugacy classes of $G$. Suppose $G$ fixes $k$ elements and has $m$ orbits of length 2 in its action on $\operatorname{Proj}\left(A, \alpha_{A}\right)$. Then $|A|=k+2 m$, and it follows from 1.8 of [4] that $k+m=z+\frac{r}{4}+\frac{t}{2}$. Thus $k=z-\frac{r}{2}$, and so $z \geq \frac{r}{2}$. Now $\left|Z\left(T_{i}\right)\right|=z+t_{i}$ and $U \leq Z\left(T_{i}\right)$, so that $z$ divides $t_{i}$ for $1 \leq i \leq 3$. Thus $z$ divides $t$ and hence $r$. We conclude that $r=0, z$, or $2 z$.

Suppose $r=0$. Then as in Case 1 we may now show that $T_{i}^{\prime}$ has order 2 for $1 \leqslant i \leqslant 3$. So $z+t_{1}=t_{2}+t_{3}, z+t_{2}=t_{1}+t_{3}, z+t_{3}=t_{1}+t_{2}$, and hence adding we obtain that $t=3 z$. Thus $[A: U]=4$.

Suppose now $r>0$. Then $\left|Z\left(T_{1}\right)\right|=z+t$. If $r=2 z$, then $|A|=3 z+t$. So $z+t$ divides $2 z$, and since $z$ divides $t$ we conclude that $t=z$. Thus $[A: U]=4$. Finally if $r=z$, then we obtain similarly that $z+t$ divides $z$, which is impossible.

We have thus proved that $G / U$ has order 16 , also $G / U$ has p.r.x. $(1, \beta)$ for any cocycle $\beta$ of $G / U$ with $\inf ([\beta])=[\alpha]$ from 1.2. Now since $A$ is a more-special subgroup of $G$ of minimal index 4 and $[A: U] \neq 2$, we have that $G$ has p.r.x. $(2,1)$ but not p.r.x. $(1,1)$ by 3.5 of [8] and 2.3. Thus there exists a cocycle $\gamma$ of $G / U$ with $\inf ([\gamma])=[1]$ for which $G / U$ is of $\gamma$-central type. Finally suppose $\operatorname{Ug}$ has order 8 in $G / U$. Then $\langle U, g\rangle$ is special and has index 2 in $G$, contrary to the definition of $A$. Thus $G / U$ has exponent 2 or 4 .

We can now immediately proceed to classify $G / U$, and hence prove Theorem 2 . The proof we shall give actually contains additional information about the various cohomology classes of $G / U$.

Proof of Theorem 2. Suppose firstly that $G / U$ is abelian. Then by 3.2, $G / U$ has exponent 2 or 4 and order 16 , so $G / U \cong \mathrm{C}_{2} \times \mathrm{C}_{2} \times \mathrm{C}_{2} \times \mathrm{C}_{2}$, or $\mathrm{C}_{4} \times \mathrm{C}_{4}$, or $\mathrm{C}_{4} \times \mathrm{C}_{2} \times \mathrm{C}_{2}$. 
Suppose $G / U \cong \mathrm{C}_{2} \times \mathrm{C}_{2} \times \mathrm{C}_{2} \times \mathrm{C}_{2}$. The set of skew-symmetric $4 \times 4$ matrices over $\mathbb{Z}_{2}$ consists of 1 matrix of rank 0,28 of rank 2, and 35 of rank 4 . These correspond as in the proof of Theorem 1 to 1,28 , and 35 cohomology classes of $G / U$ for which $G / U$ has minimal p.r.x. 0,1 , and 2 respectively.

Suppose $G / U \cong \mathrm{C}_{4} \times \mathrm{C}_{4}$. Then $M(G / U) \cong \mathrm{C}_{4}$. Now consider inf: $M(G / U) \rightarrow M(G)$. We have that $[\alpha]=\inf ([\beta])$ for some $[\beta] \in M(G / U)$, and so $\operatorname{ker}(\inf )$ must be of order 2 , since $G$ does not have p.r.x. $(1,1)$. Thus $[\beta]$ has order 4 , and $G / U$ is of $\beta$-central type, contrary to 3.2 (ii).

Finally suppose $G / U \cong \mathrm{C}_{4} \times \mathrm{C}_{2} \times \mathrm{C}_{2}$. Then by 5.4 of [12] the set of elements which are $\gamma$-regular for all cocycles of $G / U$ is isomorphic to $\mathrm{C}_{2}$. Thus in this case $G / U$ is not of $\gamma$-central type for any cocycle $\gamma$ of $G / U$, contrary to 3.2(iii). Alternatively Lemma 2 of [1] also gives this result.

Now suppose $G / U$ is non-abelian. Then again since $G / U$ has exponent 4 and order 16 we have that $G / U$ is isomorphic to one of the following five (non-isomorphic) groups: 1. $\mathrm{D}_{4} \times \mathrm{C}_{2} ; 2$. R, as in the statement of Theorem $2 ; 3 . \mathrm{Q} \times \mathrm{C}_{2}, \mathrm{Q}$ the quaternion group; 4 . $\left\langle x, y, z: x^{4}=y^{2}=z^{2}=1, x y=y x, z x=x z, y z=z x^{2} y\right\rangle ; 5 . C_{4} \ltimes C_{4}$.

In Case $1, M\left(D_{4} \times C_{2}\right) \cong C_{2} \times C_{2} \times C_{2}$ from p. 378 of [13]. Here we may consider normal cocycles $\gamma$ of $D_{4} \times C_{2}$ as in Proposition 1 of [13], and show that the elements of order 4 in $\mathrm{D}_{4}$ are $\gamma$-regular for exactly 6 classes $[\gamma]$ of $M\left(\mathrm{D}_{4} \times \mathrm{C}_{2}\right)$. For these classes $D_{4} \times C_{2}$ cannot be of $\gamma$-central type and so must have p.r.x. $(1, \gamma)$. It follows that $\left.D_{4} \times C_{2}\right)$ has p.r.x. 1 for 6 cohomology classes of $D_{4} \times C_{2}$, and has minimal p.r.x. 2 for the remaining 2 classes.

In Case 2, $M(\mathrm{R}) \cong \mathrm{C}_{2} \times \mathrm{C}_{2}$ from p. 378 of [13], and from [5] $\mathrm{R}$ has p.r.x. 1 for 3 cohomology classes of $\mathrm{R}$ and has minimal p.r.x. 2 for the remaining class.

In Cases 3, 4, or 5 we may consider groups of order 32 with centre of order 2 (see [14]), and we conclude by using 3.5 of [12] that $G / U$ is not of $\gamma$-central type for any cocycle $\gamma$ of $G / U$, contrary to 3.2 (iii).

Finally we give examples to show that groups satisfying Theorem 2 do exist, noting that examples satisfying Theorem 1 have already been given on p. 456 of [8].

EXAMPLES 1. Let $G$ be either of the extra-special 2-groups of order 32. Then $G$ has an ordinary character of degree 4 by V.16.14 of [6]. We indicated at the end of Section 2 how to show that $G$ has p.r.x. $(1, \alpha)$ for 10 cohomology classes $[\alpha]$ out of the 32 in $M(G)$. Also for any such class $[\alpha], U=Z(G)$, so that $G / U$ is elementary abelian of order 16 .

2. Let $G$ be either of the groups $G_{1}, G_{2}$ of order 32 described in [5]. Then from [5] $G / Z(G) \cong \mathrm{R}$, and $G$ has both an ordinary character of degree 4 and p.r.x. $(1, \alpha)$ for the unique non-trivial cohomology class of $G$. Also $U=Z(G)$ for $[\alpha]$.

3 . Let $G$ be either of the two groups of order 32 with $G / Z(G) \cong \mathrm{D}_{4} \times \mathrm{C}_{2}$. Then $G^{\prime}$ is cyclic of order 4 from [14]. Also $G$ has an ordinary character of degree 4 by using 3.5 of [8], or since $G$ has 11 conjugacy classes from [14]. It follows that the kernel of the inflation homomorphism from $G / Z(G)$ into $G$ has order 2. Now from the proof of Theorem 2 exactly four classes of $M\left(\mathrm{D}_{4} \times \mathrm{C}_{2}\right)$ will inflate to give two classes $[\alpha]$ of $M(G)$ for which $G$ has p.r.x. $(1, \alpha)$. Lastly $U=Z(G)$ for both such $[\alpha]$.

\section{REFERENCES}

1. F. R. DeMeyer and G. J. Janusz, Finite groups with an irreducible representation of large degree. Math. Z. 108 (1969), 145-153. 
2. R. J. Higgs, On the degrees of projective representations. Glasgow Math. J. 30 (1988), $133-135$.

3. R. J. Higgs, Groups whose projective character degrees are powers of a prime. Glasgow Math. J. 30 (1988), 177-180.

4. R. J. Higgs, Projective characters of degree one and the inflation-restriction sequence. $J$. Austral. Math Soc. (Series A) 46 (1989), 272-280.

5. P. N. Hoffman and J. F. Humphreys, Non-isomorphic groups with the same projective character tables. Comm. Algebra 15 (1987), 1637-1648.

6. B. Huppert, Endliche Gruppen. I. Die Grundlehren der mathematischen Wissenschaften in Einzeldarstellungen 134, (Springer-Verlag, Berlin, Heidelberg, New York, 1967).

7. I. M. Isaacs, Character theory of finite groups. (Pure and Applied Mathematics, a series of monographs and textbooks; 69), (Academic Press, New York, London, 1976).

8. I. M. Isaacs and D. S. Passman, Groups whose irreducible representations have degrees dividing $p^{e}$. Illinois J. Math. 8 (1964), 446-457.

9. G. Karpilovsky, Projective representations of finite groups. (Monographs and Textbooks in Pure and Applied Mathematics; 94), (Marcel Dekker, New York, Basel, 1985).

10. G. Karpilovsky, The Schur multiplier. (London Mathematical Society monographs; new ser., 2), (Oxford University Press, Oxford, New York, 1987).

11. H. N. Ng, Degrees of irreducible projective representations of finite groups. J. London Math. Soc. (2) 10 (1975), 379-384. 43-50.

12. E. W. Read, On the centre of a representation group. J. London Math. Soc. (2) 16 (1977),

13. K. I. Tahara, On the second cohomology groups of semidirect products. Math $Z$. 129 (1972), 365-379.

14. A. D. Thomas and G. V. Wood, Group tables. (Shiva mathematics series; 2), (Shiva, Orpington, 1980).

Department of Mathematics

University College, Belfield,

Dublin 4, Ireland. 